\title{
Molecular cloning, structural and expression profiling of DIRan genes during somatic embryogenesis in Dimocarpus longan Lour.
}

\author{
Zhizhen Fang, Chengchun Lai, Yaling Zhang and Zhongxiong Lai
}

\begin{abstract}
To clone and examine expression profiles of DIRan genes during somatic embryogenesis in Dimocarpus longan Lour. Thirty cDNA sequences and two genomic sequences encoding DIRan proteins were isolated from longan embryogenic cultures. Structural analysis of DIRan genes revealed that the longan Ran gene family is more expanded than that of Arabidopsis. Expression analysis of D/Ran genes during somatic embryogenesis uncovered a high abundance of DIRan genes in early embryogenic cultures and heart- and torpedo-shaped embryos. The expression of DIRan genes in embryogenic calli was affected by exogenous 2,4-dichlorophenoxyacetic acid treatment. DIRan is involved in 2,4-D induced somatic embryogenesis and development of somatic embryos in longan.
\end{abstract}

Keywords: Cloning, Dimocarpus longan, Gene expression, Ras-related nuclear protein, Somatic embryogenesis

\section{Background}

Ras-related nuclear protein (Ran) is a highly conserved, small GTPase family that is essential to multiple cellular processes in eukaryotes (Clarke and Zhang 2008). The roles of Ran have been extensively researched and well documented in animals. In contrast, little is known about Ran in plants.

Plant Ran proteins share high homology and perform similar functions in the regulation of mitotic progress with their counterparts in yeast and animals (Lü et al. 2011; Lee et al. 2008; Wang et al. 2006; Zang et al. 2010). Furthermore, Ran is involved in mediating responses to external stimuli, such as heat, salt and drought stresses (Ferreira et al. 2006; Jiang et al. 2007; Xu and Huang 2008, 2010; Yoshimura et al. 2008; Zang et al. 2010). Inhibition expression of OsRan2 in rice leads to pleiotropic developmental abnormalities (Chen et al. 2011; Zang et al. 2010). These results suggest that Ran is crucial to plant growth and development.

\footnotetext{
*Correspondence: Laizx01@163.com

Institute of Horticultural Biotechnology, Fujian Agriculture and Forestry University, 15 Shangxiadian Road, Cangshan District, Fuzhou 350002, Fujian, China
}

Longan (Dimocarpus longan Lour.), an evergreen fruit tree of great commercial value, is distributed in subtropical and tropical countries (Matsumoto 2006; Zheng et al. 2009). Longan embryo development is of great scientific interest because of its role in fruit quality and yield. The developmental regulation of Ran during the middle stage of longan somatic embryogenesis (SE) implies a role for Ran in this process (Fang et al. 2011). Furthermore, Ran has been proposed as a target for breeding and production improvement in longan (Fang et al. 2014) because of its role in delaying flowering and enhancing cold tolerance in other plants (Chen et al. 2011; Wang et al. 2006). Nevertheless, cloning and characterization of longan Ran has not yet been reported.

In this study, $30 \mathrm{cDNA}$ sequences and two genomic sequences encoding DlRan proteins were isolated. We analyzed the structures of DlRan genes, and investigated their expression profiles during SE and under exogenous 2,4-dichlorophenoxyacetic acid (2,4-D) treatment. On the basis of our results, we propose that DIRan is involved in cell division during longan SE and participates in 2,4-D-induced SE through signal transduction.

\section{罂 Springer}

(C) 2016 Fang et al. This article is distributed under the terms of the Creative Commons Attribution 4.0 International License (http://creativecommons.org/licenses/by/4.0/), which permits unrestricted use, distribution, and reproduction in any medium, provided you give appropriate credit to the original author(s) and the source, provide a link to the Creative Commons license, and indicate if changes were made. 


\section{Methods \\ Plant materials}

The establishment and maintenance of our longan embryogenic callus line "Honghezi" was described in Lai et al. (2000). The synchronization of embryogenic cultures at different developmental stages was carried out as described previously (Fang et al. 2014). All cultures were kept in dark conditions at $25 \pm 1{ }^{\circ} \mathrm{C}$.

\section{RNA extraction}

Total RNA was extracted from embryogenic cultures using TriPure Isolation Reagent (Roche Molecular Biochemicals, Basel, Switzerland) and then treated with DNase I (Takara, China) to remove genomic DNA.

\section{$5^{\prime}$ and $3^{\prime}$ rapid amplification of CDNA ends (RACE)}

A 469-bp cDNA fragment of DlRan (Ran fragment 1) was obtained by reverse-transcription PCR with degenerate primers (RanF1 and RanR1) generated according to mass spectrographic analysis results in our previous study (Fang et al. 2011). $5^{\prime}$ and $3^{\prime}$ RACE were performed to generate full-length gene transcripts. The $3^{\prime}$ RACE was performed using a First-Strand cDNA synthesis kit (Fermentas). 123 '-ends of DlRan cDNAs were obtained using specific primers designed from Ran fragment 1 (Table 1 ). Multiple alignment of these $3^{\prime}$ ends indicated the existence of DlRan homologs. A specific primer, RanR2, was designed according to the isolated $3^{\prime}$ ends, and a new DlRan fragment (DlRan fragment 2) was obtained using RanF1 and RanR2. Primers RanF8 and RanF9 were generated according to DlRan fragments 1 and 2 and used

Table 1 Specific primers used for $3^{\prime}$ and $5^{\prime}$ RACE and corresponding products

\begin{tabular}{ll}
\hline Specific primers & Products \\
\hline $\begin{array}{l}\text { Outer primer: RanF2 } \\
\text { Nested primer: RanF3 }\end{array}$ & Ran3'-1, Ran3'-2 \\
Outer primer: RanF4 & Ran3'-3, Ran3'-4, Ran3'-5, Ran3'-6, Ran3'-7, \\
Nested primer: RanF5 & Ran3'-8, Ran3'-9, Ran3'-10, Ran3'-11, Ran3'-12 \\
Outer primer: RanF8 & Ran3'-13, Ran3'-14, Ran3'-15 \\
Nested primer: RanF9 & \\
Outer primer: RanR3 & Ran5'-1, Ran5'-2, Ran5'-3, Ran5'-4, Ran5'-5 \\
Nested primer: RanR4 & \\
Outer primer: RanR5 & Ran5'-6, Ran5'-7, Ran5'-8, Ran5'-9, Ran5'-10, \\
$\begin{array}{l}\text { Nested primer: RanR6 } \\
\text { Outer primer: RanR7 }\end{array}$ & Ran5'-11 \\
Nested primer: RanR8 & \\
Outer primer: RanR9 & Ran5'-13, Ran5'-14, Ran5'-15 \\
Nested primer: RanR10 & \\
Outer primer: RanR12 & Ran5'-16, Ran5'-17 \\
Nested primer: RanR13 & \\
Outer primer: RanR11 & Ran5'-18 \\
Nested primer: RanR13 & \\
\hline
\end{tabular}

for $3^{\prime}$ RACE, yielding three additional DlRan cDNA $3^{\prime}$ ends (Table 1). A $5^{\prime}$ RACE was performed using a GeneRacer kit (Invitrogen). Specific primers were designed according to the isolated DlRan fragments and $3^{\prime}$-RACE products of DlRan and used for $5^{\prime}$ RACE. Primers and corresponding $5^{\prime}$-RACE products are indicated in Table 1. For amplification of full-length DlRan cDNAs, gene-specific primers were generated according to the DlRan $5^{\prime}$ and $3^{\prime}$ ends, with cDNAs synthesized from the GeneRacer kit used as templates. Specific primers used are listed in Table 2 and Additional file 1: Figure S1.

\section{DNA extraction and isolation of genomic DNA encoding DIRan}

Total genomic DNA was isolated from longan embryogenic calli with a Plant Genomic DNA kit (Tiangen, China). A 2389-bp DlRan DNA sequence was obtained using specific primers (RanF18 and RanR29; Table 2) and Takara LA Taq (Takara) and was designated as DlRan3A (GenBank accession no. JQ775539). The genomic sequence of $D$ Ran3B (JQ279697) has been characterized previously (Fang et al. 2013).

\section{Quantitative real-time PCR analysis}

cDNAs were synthesized with random primers and Oligo dT Primer using a SYBR ExScript kit (Takara). Real-time PCR amplifications were performed on a Lightcycler 480 system (Roche Applied Science, Switzerland) in 20- $\mu$ l total volumes containing $10 \mu \mathrm{l}$ of $2 \times$ SYBR Premix Ex Taq II (Takara), $1 \mu \mathrm{l}$ cDNA (1:10 dilution), and $0.4 \mu \mathrm{l}$ of each $0.20-\mu \mathrm{M}$ primer. PCR conditions were as follows: denaturation at $95{ }^{\circ} \mathrm{C}$ for $30 \mathrm{~s}$, followed by 40 cycles of $95^{\circ} \mathrm{C}$ for $5 \mathrm{~s}, 60{ }^{\circ} \mathrm{C}$ for $30 \mathrm{~s}$ and $72{ }^{\circ} \mathrm{C}$ for $30 \mathrm{~s}$. Reactions were run in triplicate. $E F-1 a$ and $F e-S O D$, the most stable genes selected by Lin and Lai (2010), were used as endogenous controls. Expression data were analyzed with geNORM (version 3.5) (Vandesompele et al. 2002). The high sequence similarity among isolated DlRan transcripts made it very difficult to design specific primers to detect their expression. We found that the identified DlRan transcripts could be divided into two types, N (asparagine) and D (aspartic acid), based on the tenth residue in their predicted amino acid sequences. Specific primers based on the $5^{\prime}$-end proximal region of these $\mathrm{N}$ and D DlRan transcript sequences (Additional file 2: Figure S2) were designed and used for qRT-PCR analyses. Primer pairs used for qRT-PCR analyses are listed in Table 3.

\section{Treatment of embryogenic calli with 2,4-D}

Embryogenic calli cultured on M0 medium (MurashigeSkoog basal salts, $2 \%$ sucrose and $6 \mathrm{~g} / \mathrm{L}$ agar, pH 5.8) supplemented with $1 \mathrm{mg}$ 2,4-D/l were transferred and maintained for $24 \mathrm{~h}$ on $\mathrm{M} 0$ medium or M0 medium supplemented with 
Table 2 Primers used in this study

\begin{tabular}{|c|c|c|c|}
\hline Name & Primer sequences $\left(5^{\prime}-3^{\prime}\right)$ & Name & Primer sequences $\left(5^{\prime}-3^{\prime}\right)$ \\
\hline RanF1 & GTNGGNGAYGGNGGNACNGG & RanR7 & CACCAGAGGAGCACAAAAAGCAGCAT \\
\hline RanF2 & CGTTTCTACTGCTGGGATAC & RanR8 & CTGCAACTGTTCTCTATTCAAATGTGT \\
\hline RanF3 & CTGCCAAGAGCAACTACAAT & RanR9 & TGTTCATCAACCCCAACTCCAACAAT \\
\hline RanF4 & CAGGAGAAGTTTGGTGGTCT & RanR10 & CAATCACACAATTCCCCATCCTGCT \\
\hline RanF5 & GATGTTACTGCTCGCTTGAC & RanR11 & AACNTGCTTNGCNTTCACTTGCCT \\
\hline RanF6 & CTCTGCGGAAACAARGTTGATGT & RanR12 & CANACCCNGCAAAGATNACNGTG \\
\hline RanF7 & GAARCCTTTCTTGTACCTTGCC & RanR13 & TGATNATNGCACATTGCCCATGGAT \\
\hline RanF8 & CTCTGCGGAAACAARGTTGATGT & RanR14 & TTTATGAGGCAACACTGGTTCAG \\
\hline RanF9 & GAARCCTTTCTTGTACCTTGCC & RanR15 & CCCCCTTTTTTTCCATGCAAATT \\
\hline RanF10 & CAAGACCAAAAGCTCTCCCTCTAAT & RanR16 & CCCCCCCTTTTTTTTTTAGGAG \\
\hline RanF11 & CGCTCTCAGAACCAAACCAAGAAG & RanR17 & CCCCTTTTTTTACGGAGCAAC \\
\hline RanF12 & GGTGCTTATTGATACATTTCTCC & RanR18 & CCCCTTGAAA ACCAGATAAA ATG \\
\hline RanF13 & САCTCTAATTGCCTTCCTACTTCGT & RanR19 & CCCCCTTTTTTTTTGGTATGTAAG \\
\hline RanF14 & GGCAGCAGAGAGAGAGAATC & RanR20 & CCCCCCTTTTTTTTAACAAGACC \\
\hline RanF15 & GATTGGCTGTTGTTTTGAAGAAG & RanR21 & СCCCTTTTTTTATCCTCAACACC \\
\hline RanF18 & CAAGACCAAAAGCTCTCCCTCT & RanR22 & CCCCCCCTTTTTTCAGATAATAT \\
\hline RanR1 & GRTCNCCNGCNAGYTTNCGNGC & RanR23 & СССССТTTTTATACTCAACTATC \\
\hline RanR2 & GCATCATCATCGTCATCTGG & RanR24 & TCCCCATCCTGCTGTTTTACTCGA \\
\hline RanR3 & CCTGTGGAATGTAACCTGCT & RanR25 & CCCCCCTTTTTTTTTTTTTTTAGGAGAA \\
\hline RanR4 & СCTTCACTTGCCTATTCCTC & RanR26 & CGGAGCAACGCTTAAAACATCCTACA \\
\hline RanR5 & GTCAAGCGAGCAGTAACATC & RanR29 & CAGCGTAGGGGGAGCCGAATGAAT \\
\hline RanR6 & AGACCACCAAACTTCTCCTG & RanR30 & CCAGCCTGCAACTGTTCTCTATTCA \\
\hline $5 P$ & CGACTGGAGCACGAGGACACTGA & AUAP & GCCACGCGTCGACTAGTAC \\
\hline $5 \mathrm{NP}$ & GGACACTGACATGGACTGAAGGAGTA & & \\
\hline
\end{tabular}

either $0.5,1.5$ or $2.0 \mathrm{mg} / \mathrm{l}$ of 2,4-D. All samples were frozen in liquid nitrogen after harvesting and stored at $-80^{\circ} \mathrm{C}$.

\section{Bioinformatics analysis}

Predicted protein sequences were analyzed and theoretical isoelectric points (pIs) and mass values of mature peptides were calculated using the PeptideMass program (http://us.expasy.org/tools/peptidemass.html). Amino acid sequence alignment was performed using DNAMAN software. A phylogenetic tree of Ran proteins was constructed using MEGA5 software.

Table 3 Primers used for qRT-PCR analysis

\begin{tabular}{ll}
\hline Specific primer & Primer sequences $\left(\mathbf{5}^{\prime} \mathbf{-} \mathbf{3}^{\prime}\right)$ \\
\hline N type DIRans & Forward: AAGGACAGCTCTCATGGCTTTGC \\
& Reverse: TGCCTCCATCACCGACGATGAC \\
D type DIRans & Forward: TAGTGATCGTCGGCGATGGTGG \\
& Reverse: TGCAGTGTCCCAGCAATAGAAGCG \\
Fe-SOD & Forward: GGTCAGATGGTGAAGCCGTAGAG \\
& Reverse: GTCTATGCCACCGATACAACAAACCC \\
EF-1a & Forward: GATGATTCCCACCAAGCCCAT \\
& Reverse: GGGTCCTTCTTCTCAACACTCT \\
\hline
\end{tabular}

\section{Results}

\section{Cloning of DIRan cDNAs from torpedo-shaped somatic embryos of longan}

Fifteen $3^{\prime}$ ends of DlRan genes were obtained through $3^{\prime}$ RACE. Alignment of these $3^{\prime}$ ends indicated the existence of sequence polymorphism in DlRan gene open reading frames (ORFs) and $3^{\prime}$ untranslated regions (UTRs) (Additional file 3: Figure S3). $185^{\prime}$ ends of DlRan genes were obtained using RNA ligase-mediated RACE (Additional file 4: Figure S4). Using primers designed from the isolated $5^{\prime}$ and $3^{\prime}$ ends, we isolated $30 \mathrm{DlRan}$ transcripts from torpedo-shaped somatic embryos in longan and deposited their sequences in GenBank (Table 4).

\section{Sequence analyses and molecular characterization of DIRan genes}

Sequence analysis indicated that all of the isolated DlRan transcripts contained a 663-bp ORF. The $3^{\prime}$ UTRs of the isolated DlRan transcripts lack the typical AATAAA polyadenylation signal. The isolated DlRan cDNAs were divided into nine groups according to their ORF sequences (Fig. 1). DlRan3As, DlRan3Bs, DlRan3C-1, DlRan3C-2, DlRan3C-3, DlRan3Ds, DlRan3E-1, DlRan3F-1 and DlRan3G-1 had unique ORFs (Fig. 1). 
Table 4 GenBank accession numbers of Ran cDNAs and primer pairs used for their amplifications

\begin{tabular}{lll}
\hline Name & Accession no. & Primer pairs (forward/reverse) \\
\hline DIRan3A-1 & JF461272 & RanF10/RanR14 \\
DIRan3A-2 & JF461273 & RanF10/RanR15 \\
DIRan3A-3 & JF461274 & RanF10/RanR16 \\
DIRan3A-4 & JF461275 & RanF10/RanR17 \\
DIRan3A-5 & JF461276 & RanF10/RanR18 \\
DIRan3A-6 & JF461277 & RanF10/RanR19 \\
DIRan3A-7 & JF461278 & First PCR: RanF10/3P \\
& & Nested PCR: RanF11/3NP \\
DIRan3A-8 & JF461279 & First PCR: RanF10/3P \\
& & Nested PCR: RanF11/3NP \\
DIRan3A-9 & JF461280 & First PCR: RanF10/3P \\
DIRanA-10 & JF461281 & Nested PCR: RanF11/3NP \\
DIRan3A-11 & JF461282 & Nested PCR: RanF11/3NP \\
& & First PCR: RanF10/3P \\
DIRan3A-12 & JQ861699 & Nested PCR: RanF11/3NP \\
DIRan3A-13 & JQ775533 & Nested PCR: 5NP/RanR26 \\
DIRan3A-14 & JQ775532 & RanF12/RanR24 \\
DIRAN3B-1 & HM773390 & RanF12/RanR24 \\
DIRan3B-2 & JF461283 & RanF18/RanR20 \\
DIRan3B-3 & JF461284 & RanF18/RanR21 \\
DIRan3B-5 & JF461286 & RanF18/RanR14 \\
DIRan3B-6 & JF461287 & RanF13/RanR21 \\
DIRan3B-7 & JF461288 & RanF13/RanR22 \\
DIRan3B-8 & JQ775530 & RanF13/RanR14 \\
DIRan3B-9 & JQ775531 & RanF14/RanR30 \\
DIRan3C-1 & JF461289 & RanF14/RanR30 \\
DIRan3C-2 & JF461290 & RanF13/RanR23 \\
DIRan3C-3 & JF461291 & RanF13/RanR23 \\
DIRan3D-1 & JF461292 & RanF13/RanR23 \\
DIRan3D-2 & JF461293 & RanF13/RanR17 \\
DIRan3E-1 & JF461294 & RanF10/RanR20 \\
DIRan3F-1 & JQ775527 & RanF10/RanR20 \\
DIRan3G-1 & JQ775528 & RanF10/RanR20 \\
\hline & & \\
\hline
\end{tabular}

Sequence alignment showed that the first half of sequences of DlRan3D-1, DlRan3C-1,DlRan3C-2 and DlRan3C-3 were identical to that of DlRan3B-1, while the second half of sequences of these cDNAs were identical to that of DlRan3A-1. In contrast, the first half of DlRan3E-1 and DlRan3G-1 sequences were identical to DIRan3A-1, and the second half of sequences of these cDNAs were identical to that of DlRan3B-1. One fragment of DlRan3F-1 was identical to neither DlRan3A-1 nor DlRan3B-1 (Fig. 1). These results prompted us to explore whether the transcripts identified in the present study were alternative spliced isoforms produced by the same gene or were instead transcribed from different genes.

To determine exon and intron organization of DlRan cDNAs, we try to isolate genomic sequences of DlRan genes and only 2 DlRan sequences (DlRan $3 A$ and $D l R a n 3 B$ ) were obtained. The comparative analysis of DlRan genomic and CDNA sequences indicated that DlRan3A-1-DlRan3A-14 was derived from DlRan3A and that DlRan3B-1-DlRan3B-3 and DlRan3B-5-DlRan3B-9 were derived from $D l R a n 3 B$. As indicated in Fig. 2, both $D l R a n 3 A$ and $D l R a n 3 B$ contained 8 exons. Interestingly, the first half of the sequences of DlRan $3 D-1$, DlRan3C-1, DlRan3C-2 and DlRan3C-3 were identical to the genomic sequence of $D l R a n 3 B$, while the second half of these cDNA sequences were identical to the genomic sequence of DlRan3A (Fig. 2). In contrast, the first half of sequences of DlRan3E-1 and DlRan3G-1 were identical to the genomic sequence of $D l R a n 3 A$, whereas the second half of these cDNA sequences was identical to the genomic sequence of DlRan3B (Fig. 2). Finally, the sequence of DlRan3F-1 was inconsistent with either DlRan3A or DlRan3B. Our results suggest that these transcripts were encoded by different $D l R a n$ genes rather than representing alternative spliced products from the same gene, thereby implying the existence of multiple Ran genes in the longan genome.

All of the isolated DlRan transcripts encoded seven predicted polypeptides of 221 amino acid residues with similar calculated molecular masses and predicted pIs (Table 5). It is noteworthy that DlRan3C-1, DlRan3C-2 and DlRan3C-3, which contain different ORFs, encoded the same protein. The modulation of protein expression via alteration of mRNA secondary structure has been demonstrated to involve the usage of synonymous codons (Nackley et al. 2006). We therefore used Mfold (Zuker 2003) to predict the secondary structures of the ORFs of these transcripts, which demonstrated that the Gibbs free energy for DlRan $3 C-2$ and DlRan $3 C$-3 was lower than that for DlRan3C-1.

As shown in Additional file 5: Figure S5, alignment analysis revealed that the predicted DlRan proteins are highly identical to the identified peptides in our previous study (Fang et al. 2011). This result indicates that the predicted proteins were orthologs of the identified protein. DIRan members are highly similar to one another, differing by a total of only nine amino acids. Multiple sequence alignment indicated that the DIRan proteins share a significant degree of sequence identity with Ran proteins from Arabidopsis thaliana, Medicago truncatula, Zea mays, Vitis vinifera, Allium cepa and Oryza sativa (Fig. 3). The characteristic domains of the Ran proteins that are known to be involved in GTP-binding and hydrolysis, as well as the acidic C-terminal domain 
D1Ran3B-1 ATGGCTTTGCCAAATCAGCAAACCGTTAATTACCCTAGCTTCAAGCTCGTCATCGTCGGTGATGGAGGCACT D1Ran3D-1 ATGGCTTTGCCAAATCAGCAAACCGTTAATTACCCTAGCTTCAAGCTCGTCATCGTCGGTGATGGAGGCACT D1Ran3C-2 ATGGCTTTGCCAAATCAGCAAACCGTTAATTACCCTAGCTTCAAGCTCGTCATCGTCGGTGATGGAGGCACT DIRan3C-3 1 ATGGCTTTGCCAAATCAGCAAACCGTTAATTACCCTAGCTTCAAGCTCGTCATCGTCGGTGATGGAGGCACT DIRan3C-1 ATGGCTTTGCAAATCAGCAAACCGTTAATTACCCTAGCTTCAAGCTCGTCATCGTCGGTGATGGAGGCACT DIRan3G-1 ATGGCTCTACCGAATCAGCAGACTGTTGATTATCCGAGTTTCAAGCTAGTGATCGTCGGCGATGGTGGAACT DIRan3-1 ATGGCTCACGAATCAGCAGACTGTTATTATCCAGT TCAAGCTAGTAATCGTCGGCGATGGTGGAACT DLRan3F-1 ATGGCTCTACCGAATCAGCAGACTGTTGATTATCCGAGTTTCAAGCTAGTGATCGTCGGGATGGTGGAACT

D1Ran3B-1 GGAAAGACTACCTTTGTGAAAAGGCATCTTACTGGGGAGTTCGAGAAGAAATATGAACCTACCATTGGTGTG D1Ran3D-1 GGAAAGACTACC TTTGTGAAAAGGCATCTTACTGGGGAGTTCGAGAAGAAATATGAACCTACCATTGGTGTG D1Ran3C-2 GGAAAGACTACCTTTGTGAAAAGGCATCTTACTGGGGAGTTCGAGAAGAAATATGAACCTACCATTGGTGTG GGAAAGACTACCTTTGTGAAAAGGCATCTTACTGGGGAGTTTGAGAAGAAATACGAACCCACCATTGGTGTC D1Ran3E-1 GGGAAGACTACGTTTGTGAAAAGGCATCTTACCGGAGAGTTTGAGAAGAAATACGAACCCACCATTGGTGTC D1Ran3G-1 GGGAAGACTACGTTTGTGAAAAGGCATCTTACCGGAGAGTTTGAGAAGAAATACGAACCCACCATTGGTGTC

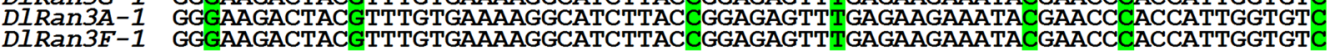

D1Ran3B-1 GAGGTTCATCCATTGGACTTCTTTACAAATTGTGGGAAAATCCGTTTCTACTGCTGGGATACAGCTGGGCAG

D1Ran3D-1 GAGGTTCATCCATTGGACTTCTTTACAAATTGTGGGAAAATCCGTTTCTACTGCTGGGATACAGCTGGGCAG

D1Ran3C-2 GAGGTTCATCCATTGGACTTCTTTACAAATTGTGGGAAAATCCGTTTCTACTGCTGGGATACAGCTGGGCAG

D1Ran3C-1 GAAGTTCATCCACTGGACTTCTTCACAAAACTGTGGGAAAATTCGCTTCTATTGCTGGGACACTGCAGGCCAA DlRan3E-1 GAAGTTCATCCACTGGACTTCTTCACAAACTGTGGGAAAATTCGCTTCTATTGCTGGGACACTGCAGGCCAA DIRan3G-1 GAATTCATCCACTGGACTTCTTCACAAACTGTGGGAAAATTCGCTTCTATTGCTGGGACACTGCAGGCCAA DIRan3A-1 GAAGTCATCCACTGGACTTCTTCACAAACTGTGGGAAAATTCGCTTCTAT TGCTGGGACACTGCAGGCCAA
DIRan3F-1 GAAGTCATCCACTGGACTTCTTCACAAACTGTGGAAAATTCGCTCTATGCTGGACACTGCAGGCAA

D1Ran3B-1 GAGAAGTTTGGTGGTCTTAGAGATGGTTACTACATCCATGGGCAATGTGCCATAATCATGTTTGATGTTACT D1Ran3D-1 GAGAAGTTTGGTGGTCTTAGAGATGGTTACTACATCCATGGGCAATGTGCCATAATCATGTTTGATGTTACT D1Ran3C-2 GAGAAGTTTGGTGTCTTAGAGATGGTTACTACATCCATGGGCATGTGCCATAATCATGTTTGATGTTACT

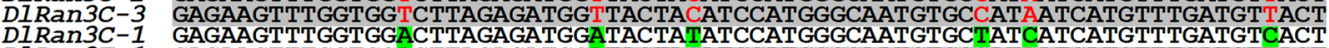
D1Ran3E-1 GAGAAGTTTGGTGGACTTAGAGATGGATACTACATCCATGGGCAATGTGCCATAATCATGTTTGATGTTACT DIRan3G-1 GAGAAGTTTGGTGGACTTAGAGATGGATACTATATCCATGGGCAATGTGCTATCATCATGTTTGATGTCACT DIRan3A-1 GAGAAGTTTGGTGGACTTAGAGATGGATACTATATCCATGGGCAATGTGCTATCATCATGTTTGATGTCACT
DIRan3F-1 GAGAAGTTGGTGGACTTAGAGATGGATACTATATCCATGGGAATGTGCTATCATCATGTTTGATGTCACT

D1Ran3B-1 GCTCGCTTGACGTACAAGAATGTTCCTACATGGCACCGTGATCTTTGCCGGGTGTGTGAAAATATACCCATT D1Ran3D-1 GCTCGCTTGACGTACAAGAATGTTCCTACATGGCACCGTGATCTTTGCCGGTGTGTGAAAATATACCCATT DIRan3C-2 GCTCGCTTGACGTACAAGAATGTTCCTACATGGCACCGTGATCTTTGCCGGTGTGTGAAAATATACCCATT $\begin{array}{ll}D 1 R a n 3 C-3 & \text { GCTCGGTTGACTTACAAGAAT GTTCCAACATGGCACAGGGATCTTTGCAGGGTCTGTGAGAACATCCCAATT } \\ D 1 \text { Ran } 3 C-1 & \text { GCTCGGTTGACTTACAAGAATGTCCAACATGGCACAGGGATCTTTGCAGGGTCTGTGAGAACATCCCAATT }\end{array}$ DIRan3E-1 GCTCGCTTGACGTACAAGAATGTTCCTACATGGCACCGTGATCTTTGCCGGGTGTGTGAAAATATACCCATT DIRan3A-1 GCTCGGTTGACTTACAAGAATGTTCCAACAAGGC DIRan3F-1 GCTCGGTTGACTTACAAGAATGTTCCAACATGGCACAGGGATCTTTGCAGGGTCTGTGAAAACATACCCATT

DIRan3B-1 GTTCTCTGCGGAAACAAAGTTGATGTGAGGAATAGGCAAGTGAAGGCAAAGCAGGTTACATTCCACAGGAAG DIRan3D-1 GTTCTCTGCGGAAACAAAGTTGATGTGAGGAATAGGCAAGTGAAGGCAAAGCAGGTTACATTCCACAGGAAG DIRan3C-2 GTTCTCTGCGGAAACAAGGTTGATGTTAAGAACAGGCAAGTGAAAGCCAAGCAAGTTACTTTCCACAGGAAA D1Ran3C-3 GTTCTCTGCGGAAACAAGGTTGATGTTAAGAACAGGCAAGTGAAAGCCAAGCAAGTTACTTTCCACAGGAAA DIRan3C-1 GTTCTCTGGGAAACAAGGTTGATTIAGAACAGGCAAGTGAAAGCCAAGCAAGTTACTTTCCACAGGAAA

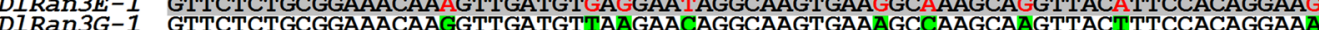

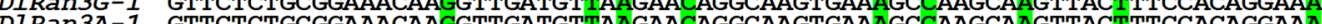
DIRan3A-1 GTTCTCTGCGGAAACAAGGTTGATGTTAAGAACAGGCAAGTGAAAGCCAAGCAAGTTACTTTCCACAGGAAA
DIRan3F-1

D1Ran3B-1 AAGAATCTGCAGTACTATGAGATCTCTGCCAAGAGCAACTACAATTTTGAGAAACCTTTCTTGTACCTTGCC DIRan3D-1 AAGAATCTGCAGTACTATGAGATCTCTGCCAAGAGCAACTACAATTTTGAGAAACCTTTCTTGTACCTTGCC AAGAACTTGCAATACTATGAGATCTCAGCAAAGAGCAATTACAACTTTGAGAAGCCTTTCTTGTACCTTGCC DIRan3C-1 AAGAACTTGCAATACTATGAGATCTCAGCAAAGAGCAATTACAACTTTGAGAAGCCTTTCTTGTACCTTGCC DIRan3E-1 AAGAATCTGCAGTACTATA DIRan3G-1 AAGAACT TGCAATACTATGAGATCTCAGCAAAGAGCAATTACAACTTTGAGAAGCCTTTCTTGTACCTTGCC DlRan3A-1
AlRan3F-1 AAGACT TGCAATACTATGAGATCTCAGCAAAGAGCAATTACAACTTTGAGAAGCCTTTCTTGTACCTTGCC D1Ran3B-1 CGCAAGCTTGCAGGGGACCCTAACATTCACTTTGTGGAGTCTCCTGCTCTTGCTCCTCCAGAAGTACACATA DlRan3D-1 CGCAAGCTTGCAGGGGACCCTAACATTCACTTTGTGGAGTCTCCTGCCCTCGCACCTCCTGAGGTTCAAATC DIRan3C-2 AGAAGCTTGCCGGGGATGCTAACTTGCATTTCGTTGAGTCTCCTGCCCTCGACCTCCTAGGTCAAATC DIRan3C-1 AGAAAGCTTGCCGGGGATGCTAACTTGCATTCGTTGAGTCTCCTGCCCTCGCACCTCCTGAGGTTCAAATC

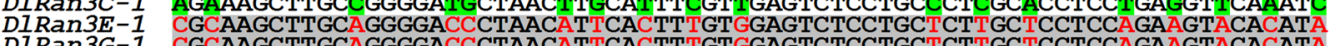
$\begin{array}{ll}D l R a n 3 G-1 & \text { CGCAAGCTTGCAGGGGACCCTAACATTCACTTTGTGGAGTCTCCTGCTCTTGCTCCTCCAGAAGTACACATA } \\ \text { DIRan3A-1 AGAAAGCTTGCCGGGGATCTAACTTGCATTTCGTTGAGTCTCCTGCCCTCGCACCTCCTGAGGTTCAAATC }\end{array}$ DIRan3A-1 AGAAAGCTTGCCGGGGATGCTAACTTGCATTTCGTTGAGTCTCCTGCCCTCGCACCTCCTGAGGTTCAAATC
DIRan3F-1 CGCAAGCTTGCGGGGGACCCTAACATTCACTTTGTGGAGTCTCCTGCTCTTGCTCCTCCAGAAGTACAAATA

D1Ran3B-1 GATTTGGCTGCCCAACAACATCATGAAGCTGAGCTTGCTGCGGCAGCTAGTCAGCCTCTTCCTGATGACGAT DIRan3C-2 GATTTGGCAGCTCAACAACAGCATGAGGCTGACTGCAAGCTGCTGCTAGTCAACCACTTCCAGATGACGAT DIRan3C-3 GATTTGGCAGCTCAACAACAGCATGAGGCTGAACTGCAAGCTGCTGCTAGTCAACCACTTCCAGATGACGAT DIRan3C-1 GATTTGGCAGCTCAACAACAGCATGAGGCTGAACTGCAAGCTGCTGCTAGTCAACCACTTCCAGATGACGAT DIRan $3 E-1$ GATTTGGCTGCCCAACAACATCATGAAGCTGAGCTTGCTGCGGCAGCTAGTCAGCCTCTTCCTGATGACGAT DIRan3G-1 GATTTGGCTGCCCAACAACATCATGAAGCTGAGCTTGCTGCGGCAGCTAGTCAGCCTCTTCCTGATGACGAT DIRan3A-1 GATTTGGCAGCTCAACAACAGCATGAGGCTGAACTGCAAGCTGCTGCTAGTCAACCACTTCCAGATGACGAT
DIRan3F-1 GATTGGCTGCCAACAACAGCATGAAGCTGAGCTTGCTGCGGCAGCTAGTCAGCCTCTTCCTGATGACGAT

DIRan3B-1 GATGACACATTTGAA

DIRan3B-1 GATGATGCATTCGAG

DlRan3C-2 GATGATGCATTCGAG

DlRan3C-3 GATGATGCATTCGAG

DIRan3C-1 GATGATGCATTCGAG

DIRan3G-1 GATGACACATTTGA

DIRan3A-1 GATGATGCATTCGAG

Fig. 1 Multiple alignments of the open reading frame sequence of DIRan genes. Sequence fragments consistent with DIRan3B-1 were indicated with grey shadow, sequence fragment of DIRan $3 F-1$ that is not consistent with DIRan3B-1 nor DIRan3A-1 were highlighted with underline, different bases among the aligned sequences are indicated by colors 


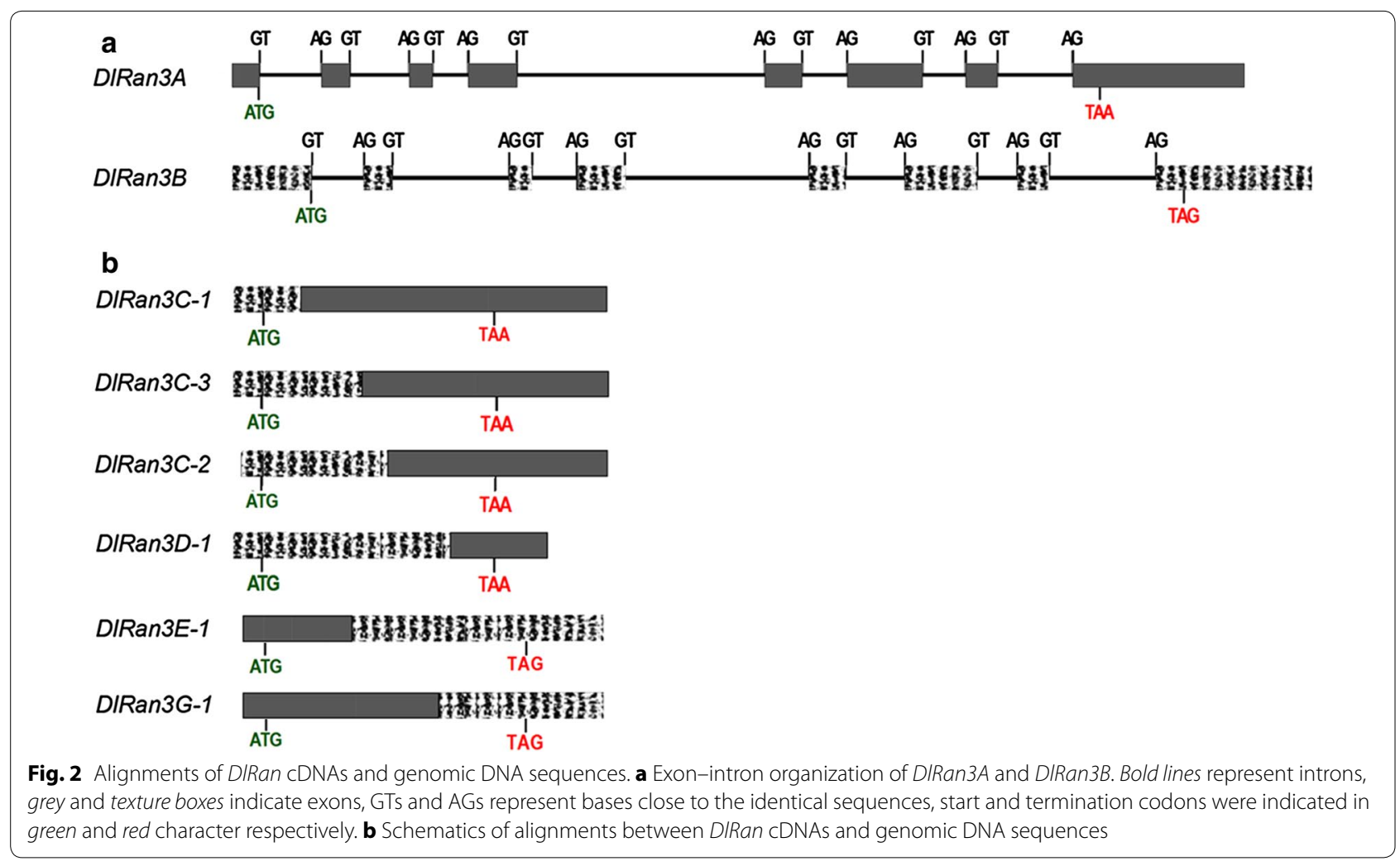

Table 5 Calculated molecular mass and predicted pl of DIRan proteins

\begin{tabular}{lll}
\hline Protein name & Molecular weight (Da) & pl \\
\hline DIRan3A & $25,106.5$ & 6.38 \\
DIRan3B & $25,150.6$ & 6.75 \\
DIRan3C & $25,105.5$ & 6.65 \\
DIRan3D & $25,159.6$ & 6.65 \\
DIRan3E & $25,151.5$ & 6.50 \\
DIRAN3F & $25,147.6$ & 6.65 \\
DIRAN3G & $25,123.5$ & 6.50 \\
\hline
\end{tabular}

and the effector-binding domain, were detected in the deduced DlRan proteins (Fig. 3). As shown in Fig. 3, the conserved sequences of these motifs were nearly identical between DlRan proteins and Ran proteins from other plant species, except for AtRan4, which has distinct functions in Arabidopsis (Vernoud et al. 2003). In the neighbor-joining phylogenetic tree based on the DIRan proteins and Ran proteins from multiple plant species, the DlRan proteins, AtRan3 and Ran3-like proteins from Glycine max and $V$. vinifera were clustered into one group (Fig. 4). These results suggest that the DIRan proteins are Ran3 homologs.

\section{Expression analysis of DIRan genes during SE in longan} We used qRT-PCR to detect abundances of DlRan transcripts at different developmental stages of longan SE. As indicated in Fig. 5, the expression profiles of two types of DlRan genes during longan SE were very similar. High levels of DlRan transcripts were detected in early embryogenic cultures and heart- and torpedo-shaped embryos. The highest levels were found in heart-shaped embryos, while the lowest were detected in globular, cotyledonary and mature embryos.

\section{The effect of 2,4-D on expression of DIRan genes in longan} embryogenic calli

2,4-D is a growth regulator commonly used in the induction of somatic embryos. However, high concentrations inhibit development of somatic embryos in longan and other plants (Aiqing et al. 2011; Lai et al. 2000). Furthermore, application of 2,4-D in various concentrations is able to synchronize SE in longan (Chen and Lai 2002). Wang et al. (2006) have demonstrated that Ran is involved in auxin signaling. $1 \mathrm{mg} 2,4-\mathrm{D} / \mathrm{l}$ is necessary to maintain longan calli at embryogenic state (Lai et al. 2000). To investigate the effect of 2,4-D on the expression of DlRan genes, embryogenic calli cultured on M0 medium supplemented with $1 \mathrm{mg} 2,4-\mathrm{D} / \mathrm{l}$ were transferred to M0 medium supplemented with different 
DIRan3A MALPNQQTVDYPSFKIVIVGDGGTGKTTFVKRHLTGEFEKKYEPTIGVEVHPLDFFTNCGKIRFYCWDTAGQEKFGGLRD

DIRan3B MALPNQQTVNY PSFKLVIVGDGGTGKTTFVKRHLTGEFEKKYEPTIGVEVHPLDFFTNCGKIRFYCWDTAGQEKFGGLRD DIRan3C MALPNQQTVNYPSFKLVIVGDGGTGKTTEVKRHLTGEFEKKYEPTIGVEVHPLDFFTNCGKIRFYCWDTAGQEKFGGLRD DIRan3D MALPNQQTVNYPSFKLVIVGDGGTGKTTEVKRHLTGEFEKKYEPTIGVEVHPLDFFTNCGKIRFYCWDTAGQEKFGGLRD DIRAN3E MALPNQQTVDYPSFKLVIVGDGGTGKTTFVKRHLTGEFEKKYEPTIGVEVHPLDFFTNCGKIRFYCWDTAGQEKFGGLRD DIRAn3F MAIPNQQTVDYPSFKIVIVGDGGTGKTTEVKRHLTGEFEKKYEPTIGVEVHPLDFFTNCGKIRFYCWDTAGQEKFGGLRD DIRAN3G MALPNQQTVDYPSFKLVIVGDGGTGKTTFVKRHLTGEFEKKYEPTIGVEVHPLDFFTNCGKIRFYCWDTAGQEKFGGLRD AsRan2 MALPNQQTVDYPFKIVIVGDGGTGKTTEVKRHLTGEFEKKYEPTIGVEVHPLDFFTNCGKIRFYCWDTAGQEKFGGLRD

MtRan

OsRan MAIPNQOTVDY PFKLVIVGDGGTGKTTEVKRHLTGEFEKKYEPTIGVEVHPLDFFTNCGKIRFYCWDTAGQEKFGGLRD MALPNQQTVDY PSFLLIVGDGGTGKTT FVKRHLTGEFEKKYEPTIGVEVHPLDFFTNCGKIRFYCWDTAGQEKFGGLRD

VvRan

ZmRan MAIPNQQTVDY SFKIVIVGDGGTGKTT EVKRHLTGEFEKKYEPTIGVEVHPLDFFTNCGKIRFYCWDTAGQEKFGGLRD

AtRan1

AtRan2 MAI PNQQ GVDY PSKLVIVGDGGTGKTT EVKRHLTGEFEKKYEPTIGVEVHPLDFSTNCGKIRFY CWDTAGQEKFD GLRD MAL PNQQTVDYPSFKLVIVGDGGTGKTT FVKRHLTGEFEKKYEPTIGVEVHPLDFFTNCGKIRFYCWDTAGQEKFGGLRD MAI PNQQTVDYPSFKIVIVGDGGTGKTT FVKRHLTGEFEKKYEPTIGVEVHPLDFFTNCGKIRFYCWDTAGQEKFGGLRD AtRan3 MALPNQQTVDY PSFKLVIVGDGGTGKTTFVKRHLTGEFEKKYEPTIGVEVHPLDFFTNCGKIRFYCWDTAGQEKFGGLRD AtRan4 MALPNQQNVDLPTFKLLIVGDGGTGKTTFLKRHLTGEFEHNTEPTLGVDIYPLDFFTNRGKIRFECWDTAGQEKYSGLKD
$* * * * * * * * * * * * * * *$ RanGAP-binding

\section{G4}

DIRan3A GYYIHGQCAIIMFDVTARLTYKNVPTWHHDLCRVCENIPIVLCGNKVDVKNRQVKAKQVTFHRKKNLQYYEISAKSNYNF DIRan3B GYYIHGQCAIIMFDVTARLTYKNVPTWHRDLCRVCENIPIVLCGNKVDVRNRQVKAKQVTFHRKKNLQYYEISAKSNYNF DIRan3C GYYIHGQCAIIMFDVTARLTYKNVPTWHRDLCRVCENIPIVLCGNKVDVKNRQVKAKQVTFHRKKNLQYYEISAKSNYNF DIRan3D GYYIHGQCAIIMFDVTARLTYKNVPTWHRDLCRVCENIPIVLCGNKVDVRNRQVKAKQVTFHRKKNLQYYEISAKSNYNF DIRAn3E GYY IHGQCAIIMFDVTARLTYKNVPTWHRDLCRVCENIPIVLCGNKVDVRNRQVKAKQVTFHRKKNLQYYEISAKSNYNF DIRAN3F GYYIHGQCAIIMFDVTARLTYKNVPTWHRDLCRVCENIPIVLCGNKVDVRKRQVKAKQVTFHRKKNLQYYEISAKSNYNF DIRan3G GYY IHGQCAIIMFDVTARLT YKNVPTWHRDLCRVCENIPIVLCGNKVDVKNRQVKAKQVTFHRKKNLQYYEISAKSNYNF AsRan2 GYYIHGQCAIIMFDVTARLTYKNVPTWHRDLCRVCENIPIVLCGNKVDVKNRQVKAKQVTFHRKKNLQYYEISAKSNYNF

MtRan

OsRan GYY IHGQCA I IMFDVTARLT YKNVPTWHRDLCRVCENIPIVLCGNKVDVKNRQVKAKQVTFHRKKNLQYYEISAKSNYNF

VvRan

ZmRan GYY I HGQCA I IMFDVTSRLT YKNVPTWHRDLCRVCENI I ILCGNKVDVKNRQVKAKQVTFHRKKNLQYYEVSAKSNYN GYY IHGQCA I IMFDVTARLT YKNVPTWHRDLCRVCENIP IVLCGNKVDVKNRQVKAKQVTFHRKKNLQYYE ISAKSNYNF

AtRan1 GYY IHGQCA I IMFDVTSRLT YKNVPTWHRDLCRVCENIP IVLCGNKVDVKNRQVKAKQVTFHRKKNLQYYEISAKSNYNF

AtRan2 GYY IHGQCA I IMFDVTARLTYKNVPTWHRDLCRVCENIPIVLCGNKVDVKNRQVKAKQVTFHRKKNLQYYEISAKSNYNF GYY IHGQCA I IMFDVTARLTYKNVPTWHRDLCRVCENIP IVLCGNKVDVKNRQVKAKQVTFHRKKNLQYYE ISAKSNYNF

AtRan3 GYY I HGQCA I IMFDVTARLT YKNVPTWHRDLCRVCENIP IVLCGNKVDVKNRQVKAKQVTFHRKKNLQYYEISAKSNYNF AtRan4 AYY I HGQCA I IMFDVTARHTYMNIDRWYRDLRRVCKNIP IVLCGNKVDVP SRQIKPKHVS YHRKKCLQYYFMSAKNNCNF

80 80 80 80 80 80 80 80 80 80 80 80 80 80 80 80 160 160 160 160 160 160 160 160 160 160 160 160 160 160 160 160

\begin{tabular}{|c|c|c|}
\hline & $\begin{array}{l}\text { Acidic tail } \\
\nabla \nabla \nabla \nabla \nabla \nabla \nabla \nabla\end{array}$ & \\
\hline DIRan3A & EKPFLYLARKLAGDANLHFVES PALAPPEVQIDIAA $Q Q Q H E A E L Q A A A S Q$ PLPDDDDDAFE. & 221 \\
\hline DIRan3B & EKPFLYLARKLAGD PNIHFVESPALAPPEVHIDLAAQQHHEAELAAAASQPLPDDDDDTFE . & 221 \\
\hline & KPFLYLARKLAGDANLHFVESPALAPPEVQIDLAA $Q Q Q H E A E L Q A A A S Q P L P D D D D D A F E$. & 221 \\
\hline DIRan3D & EKPFLYLARKLAGD PNIHFVES PALAPPEVQIDLAAQQQHEAELQAAASQPLPDDDDDAFE. & 221 \\
\hline DIRan3E & ARKLAGD PNI HFVES PALAPPEVHIDIAAQQHHEAELAAAA SQPLPDDDDDTFE . & 221 \\
\hline & EKPFLYLARKLAGD PNIHFVESPALAPPEVQIDLAAQQQHEAELAAAASQPLPDDDDDTFE. & 221 \\
\hline DIRan3G & EKPFLYLARKLAGD PNIHFVESPALAPPEVHIDLAAQQHHEAELAAAASQPLPDDDDDTFE . & 221 \\
\hline n2 & EKPFLYLARKLAGDQNLHFVESPALAPPEVHLDIAAQQQNEADLAAAAAQPLP . DDDDAFE. & 220 \\
\hline & EKPFLYLARKLAGD PNLHFVESPALAPPEVQIDLVAQQQHENELQQAANQ PLPDDDDDAFE. & 221 \\
\hline Os & EKP FLYLARKLA GD GNL HFVETPALAPPDVTIDIAAQQQ HEAELAAAAAQPLPDDDDDLIE. & 221 \\
\hline VvF & ARKLAGD PNLHFVES PALAPPEVHIDIAAQQQHEAELAAAASQPLPDDDDDAFE . & 221 \\
\hline $\mathrm{Zml}$ & EKPFLYLARKLAGDQNLHFVEAVALKPPEVQIDMALQQQHEAELAAAAAQPLPDDDDDIIE . & 221 \\
\hline AtR & EKPFLYLARKLAGD $Q N L H F V E T P A L A P P E V H I D I A D Q Q K N E A E L L Q A A A Q P L P D D D D D I F E$. & 221 \\
\hline AtRan2 & EKPFLYLARKLAGDQNLHFVESPALAPPEVHIDIAAQQQNEADLAAAAAQELPDDDDDAFE. & 221 \\
\hline AtR & EKPFLYLARKLAGDANLHFVESPALAPPEVQIDIAAQQQHEAELAAAASQPLPDDDDDTFE. & 221 \\
\hline ItRan4 & EKPFLYLARRIAGDAKLSFVES . . . . PEAQIDNLDVESLQLLTVEAGTQPLLMTRISFEFNTLSIE & 222 \\
\hline
\end{tabular}

Fig. 3 Multiple alignments of the deduced DIRan sequences with other Ran sequences. Sequences are from A. thaliana (AtRan1, NP_197501; AtRan2, NP_197502; AtRan3, NP_200330; AtRan4, NP_200319), M. truncatula (MtRan, ACJ83982), Z. mays (ZmRan, NP_001149221), V. vinifera (VvRan, XP_002284967), A. cepa (AsRan2, ABD17864) and O. sativa (OsRan, NP_001043550). Identical and similar amino acid residues among the aligned sequences are indicated by green, yellow and grey shading, respectively. Conserved GTP binding and hydrolysis domains (G1-G5) were indicated by bold lines. The effector-binding domain (RanGAP-binding) and the acidic C-terminal region (acidic tail) are indicated with asterisks and triangles, respectively 


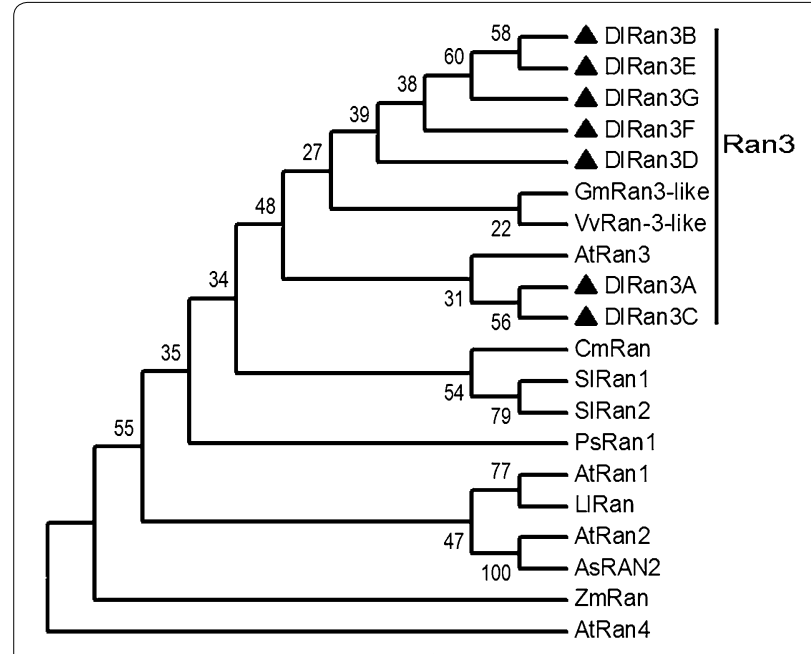

Fig. 4 Phylogenetic relationships of Ran proteins from D. longan and selected plant species. Phylogenetic and evolutionary analyses were performed using the neighbor-joining method by MEGA5 software with 1000 bootstrap replicates. A. thaliana (AtRan1, NP_197501, AtRan2, NP_197502, AtRan3, NP_200330), V. vinifera (VvRan3-like, XP_002285018), G. max (GmRan3-like, XP_003526422), Cucurbita maxima (CmRan, AEK84227), Solanum lycopersicum (SIRan1, NP_001234016, SIRan2, NP_001234023), Pisum sativum (PsRan1, ABM73376), Lepidium latifolium (LIRan, AEK78856), Allium sativum (AsRan2, ABD17865), Z. mays (ZmRan, NP_001149221)

concentrations of 2,4-D. As indicated in Fig. 6, reducing the concentration of 2,4-D gradually increased the abundance of DlRan gene transcripts. Increasing the concentration of 2,4-D to $1.5 \mathrm{mg} / \mathrm{l}$ also enhanced the accumulation of DlRan genes transcripts. In contrast, application of $2.0 \mathrm{mg} 2,4-\mathrm{D} / 1$ reduced the abundance of DlRan transcripts to levels lower than initial values.

\section{Discussion}

\section{Characterization of an expanded Ran gene family in longan}

The Ran gene family comprises a small number of genes found in different organisms, namely one member in humans and Schizosaccharomyces pombe and four in Arabidopsis (Ma 2007; Takai et al. 2001). In this study, 30 DlRan cDNAs were cloned from torpedo-shaped embryos in longan. Alignments between DlRan cDNA sequences and genomic DNA sequences suggested the existence of more Ran genes in the longan genome. Phylogenetic analysis revealed that seven deduced DIRan proteins are closely related to Ran3 from other species. Our results suggest that the longan Ran gene family is expanded compared with Arabidopsis (Ma 2007). The estimated size of the longan genome is $444 \mathrm{Mb}$ (VanBuren et al. 2011), about threefold larger than that of Arabidopsis. Nevertheless, the exact number of Ran genes in longan cannot be determined until whole

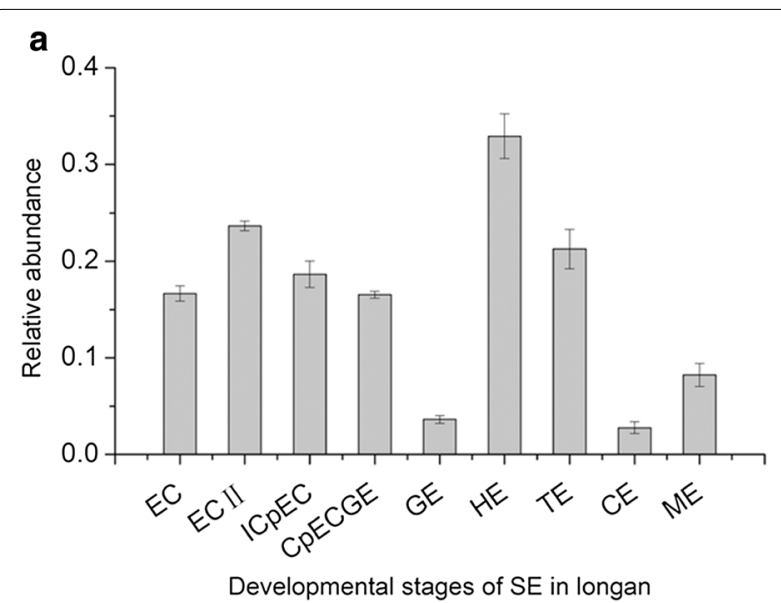

b

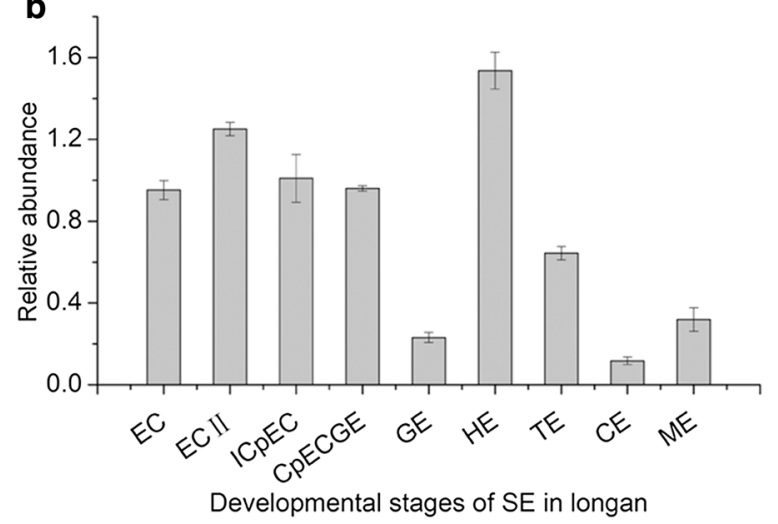

Fig. 5 Relative expression levels of D/Ran genes during longan somatic embryogenesis determined by qRT-PCR. Expression level was normalized to $\mathrm{Fe}-\mathrm{SOD}$ and $E F-1 a$. Data are mean $\pm \mathrm{SE}(\mathrm{n}=3)$. $\mathbf{a}$ Expression level of N type DIRan transcripts (DIRan3B-1-DIRan3B-9, DIRanC-1-DIRan3C-3, DIRanD-1and DIRanD-2). b Expression level of D type DIRan transcripts (DIRan3A-1-DIRan3A-14, DIRanE-1, DIRanF-1 and DIRanG-1). EC friable-embryogenic callus, EC // embryogenic callus II, ICPEC incomplete compact pro-embryogenic cultures, CPECGE compact proembryogenic cultures, GE globular embryos, HE heart-shaped embryos, TE torpedo-shaped embryos, CE cotyledonary embryos, ME mature embryos. Morphology of these embryogenic cultures has been described in previous studies (Lai et al. 2012; Lai and Lin 2013)

genome sequencing is completed. Sequence features of the longan Ran gene family that may be unique to this species and cannot be determined until all Ran genes have been isolated from the longan genome.

\section{Regulation of DIRan gene expression}

In the present study, DlRan genes were significantly upregulated at the heart-shaped embryo stage. At the torpedo-shaped embryo stage, DlRan genes were downregulated whereas the Ran protein was rapidly upregulated. Our results indicate that the expression patterns of DlRan genes were different from that of the Ran protein 

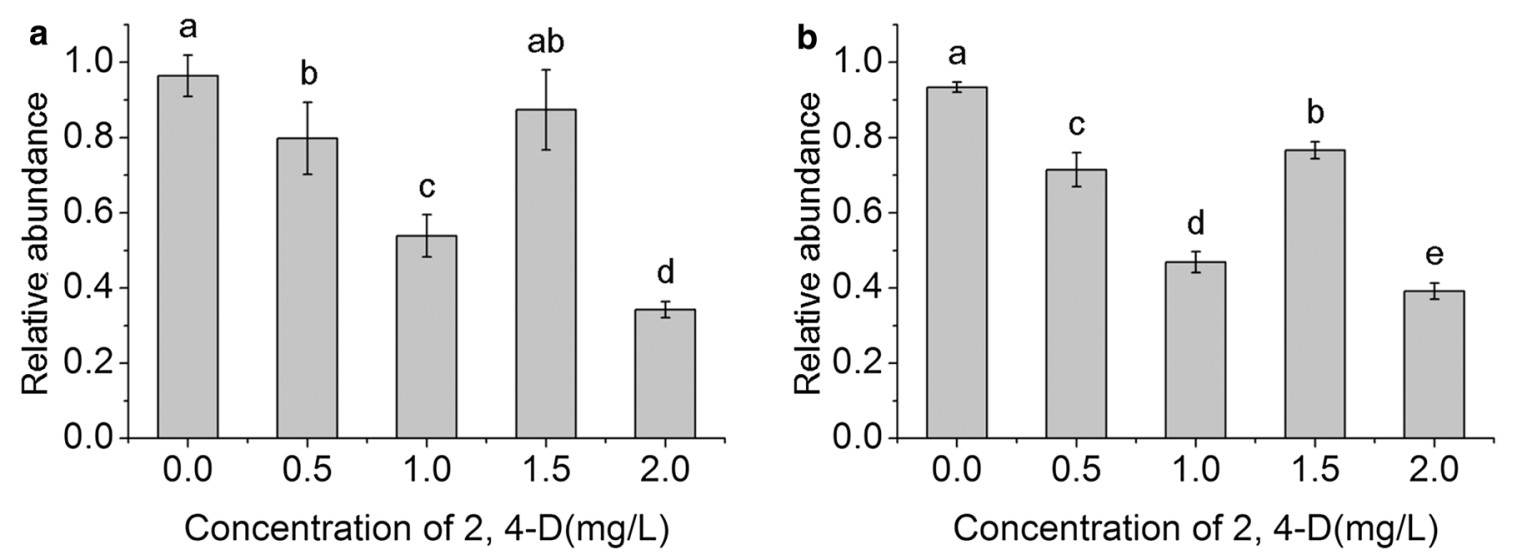

Fig. 6 Expression of DIRan genes under 2, 4-D treatment. Embryogenic calli were treated with M0 supplemented with 0.5, 1.5 and 2.0 mg/l of 2,4-D and 2,4-D free medium, respectively. RNA was extracted from embryogenic calli and analyzed by realtime PCR to determine the relative abundance of D/Ran genes. a Abundance of N type DIRan transcripts, $\mathbf{b}$ abundance of D type DIRan transcripts. Abundance was normalized to Fe-SOD and EF1a. Significance was tested by one-way ANOVA using SPSS 13.0. Different letters above the bars indicate significant differences according to the least significant difference test at $5 \%$ level. Data are mean \pm SE $(n=3)$

identified in our previous study (Fang et al. 2011; Lai et al. 2012). Discordance between protein and mRNA expression is a common phenomenon in eukaryotic cells (Skrzycki et al. 2010; Wang et al. 2010). We speculate that unidentified post-transcriptional mechanisms participate in regulation of DlRan gene expression.

We found that changes in synonymous codon usage gave rise to mRNA secondary structure alterations among DlRan3C-1, DlRan3C-2 and DlRan3C-3. Although synonymous mutations have no effect on the resulting protein sequence, the selection of synonymous codons affects the modulation of gene expression and cellular functions (Plotkin and Kudla 2011). The differential usage of synonymous codons among these transcripts may be functional, but further tests are required to confirm this hypothesis.

\section{Potential functions of DIRan genes during SE in longan}

The involvement of Ran in longan SE has been demonstrated previously (Fang et al. 2011). Our results indicated that reduction of 2,4-D concentration in the medium, which promotes initiation of somatic embryo development, enhanced DlRan gene expression. This result further supports the involvement of DlRan in longan SE. Plant Ran is involved in cell proliferation (Lü et al. 2011; Wang et al. 2006). The sequence alignment in the present study indicates that DIRan proteins are highly conserved with respect to Ran proteins from other plants, suggesting similar functionality. Our expression analysis showed that DlRan gene transcripts are more abundant during SE stages associated with active cell division. The high expression of DlRan genes observed at heart- and torpedo-shaped stages may be related to the cell proliferation that gives rise to the cotyledons and radicle. We believe that DIRan proteins may regulate mitotic progress in a manner similar to their homologs in other plants.

2,4-D was shown to alter Ran expression when applied at different concentrations. Auxin plays pivotal roles in SE. 2,4-D, the most commonly used synthetic auxin for induction of SE (Karami and Saidi 2010), affects the indole acetic acid (IAA) synthetic pathway and promotes IAA accumulation (Michalczuk et al. 1992a, b). Ectopic postembryonic expression of $L E C 2$ has been shown to induce somatic embryo formation (Stone et al. 2001). LEC2 has been proposed to induce SE by promoting auxin activity, and 2,4-D exerts effects similar to those of ectopic $L E C 2$ expression (Stone et al. 2008). Su et al. (2009) have suggested that exogenous auxin levels play an important role in determining expression patterns of WUS, a correct expression of which is essential for somatic embryo induction. 2,4-D can induce SE, but also inhibits somatic embryo development (Aiqing et al. 2011). Pan et al. (2010) found that treatment with high concentrations of 2,4-D changed the proteome of Valencia embryogenic callus. Although the mechanisms involved in induction of SE by 2,4-D and the inhibitory effect of this auxin on somatic embryo development remain to be uncovered, 2,4-D functions by altering gene expression in plant cells through signal transduction. Ran is a vital regulator of nucleocytoplasmic trafficking in plants (Meier and Somers 2011; Merkle 2011). Numerous studies have detailed the involvement of Ran in plant responses to hormonal and environmental signaling (Ferreira et al. 2006; Jiang et al. 2007; Kriegs et al. 2006; Lee et al. 2008; Mahong et al. 2012; Wang et al. 
2006; Xu and Huang 2010; Yoshimura et al. 2008). Ran is involved in auxin signaling (Wang et al. 2006) and it is unsurprising to find that Ran expression is influenced by $2,4-\mathrm{D} .1 \mathrm{mg} 2,4-\mathrm{D} / \mathrm{l}$ is necessary to maintain longan calli at embryogenic state, remove or reduce the concentration of 2,4-D initiates the development of somatic embryos. Nucleocytoplasmic transport and cell division are essential during the formation of somatic embryos. It is reasonable that the expression of Ran was enhanced by reducing the concentration of 2,4-D. Properly increasing the concentration of 2,4-D promote the proliferation of longan calli and improve the expression of Ran. However, $2 \mathrm{mg}$ 2,4-D/l inhibit the growth of longan calli and cause browning, which can explain the repression effect of $2 \mathrm{mg}$ 2,4-D/l on Ran level. Our results further support the involvement of Ran in auxin signal transduction. Zang et al. (2010) have suggested that Ran participates in abiotic response signaling by modulating the nuclear transportation of proteins and RNA. Taking the results of these studies and ours into consideration, we speculate that DlRan may participate in 2,4-D-induced SE by transmitting 2,4-D signals and may regulate the expression of embryogenesis-related genes by controlling nuclear trafficking.

In this study, $30 \mathrm{cDNA}$ and two genomic DNA sequences of DlRan genes were isolated. We also revealed the expression profiles of DlRan genes during SE and under exogenous 2,4-D treatment. Our results suggest the importance of DlRan genes in longan embryo development. Future research should focus on the elucidation of mechanisms involved in regulation of DlRan gene expression and the functions of different DlRan genes during SE in longan.

\section{Additional files}

Additional file 1: Figure S1. Schematic of RACE primer locations. Arrows indicate the locations of RACE primers.

Additional file 2: Figure S2. Location of binding sites for QRT-PCR primers.

Additional file 3: Figure S3. Alignment of $3^{\prime}$ ends of DIRan CDNAs.

Additional file 4: Figure S4. Alignment of $5^{\prime}$ ends of DIRan cDNAs.

Additional file 5: Figure S5. Multiple sequence alignment of DIRan proteins and previously identified Ran peptides.

\section{Authors' contributions}

ZF and YL conceived and designed the experiments. ZF, CL and YZ performed the experiments. ZF, $C L$ and $Z L$ analyzed the data. $Z F$ and $Z L$ wrote the paper. All authors read and approved the final manuscript.

\section{Acknowledgements}

This work was funded by the National Natural Science Foundation of China (31272149 and 31572088) and Fujian provincial Major Special Project of Agricultural science and technology (2015NZ0002-1).

\section{Competing interests}

The authors declare that they have no competing interests.

Received: 17 November 2015 Accepted: 16 February 2016

Published online: 25 February 2016

\section{References}

Aiqing J, Xueqing G, Yan Z, Yang H, Guoliang W (2011) Advances in somatic embryogenesis research of horticultural plants. Am J Plant Sci 2:727-732. doi:10.4236/ajps.2011.26087

Chen CL, Lai ZX (2002) Synchronization regulation of embryogenesis of embryogenic calli and their histological observation in longan (Dimocarpus longan Lour.). J Fujian Agric Univ (Nat Sci) 31:192-194

Chen NA, Xu Y, Wang X, Du C, Du J, Yuan M, Xu Z, Chong K (2011) OsRAN2, essential for mitosis, enhances cold tolerance in rice by promoting export of intranuclear tubulin and maintaining cell division under cold stress. Plant Cell Environ 34:52-64. doi:10.1111/j.1365-3040.2010.02225.x

Clarke PR, Zhang C (2008) Spatial and temporal coordination of mitosis by Ran GTPase. Nat Rev Mol Cell Biol 9:464-477. doi:10.1038/nrm2410

Fang ZZ, Lai ZX, Lai CC, Jiang SR (2011) Proteomics on somatic embryogenesis during the middle stage in longan. Sci Agric Sin 44:2966-2979. doi:10.3864/j.issn.0578-1752.2011.14.014

Fang ZZ, Lai CC, Zhang YL, Lin YL, Lai ZX (2013) Identification of a PTC-containing DIRan transcript and its differential expression during somatic embryogenesis in Dimocarpus longan. Gene 529:37-44. doi:10.1016/j. gene.2013.07.091

Fang ZZ, Zhang YL, Lai CC, Lai ZX (2014) Developmental regulation of Ran 3'untranslated region during somatic embryogenesis in Dimocarpus longan Lour. Sci Hortic 176:297-302. doi:10.1016/j.scienta.2014.07.023

Ferreira S, Hjernø K, Larsen M, Wingsle G, Larsen P, Fey S, Roepstorff P, Salomé Pais M (2006) Proteome profiling of Populus euphratica Oliv. upon heat stress. Ann Bot 98:361-377. doi:10.1093/aob/mcl106

Jiang Y, Yang B, Harris NS, Deyholos MK (2007) Comparative proteomic analysis of $\mathrm{NaCl}$ stress-responsive proteins in Arabidopsis roots. J Exp Bot 58:3591-3607. doi:10.1093/jxb/erm207

Karami O, Saidi A (2010) The molecular basis for stress-induced acquisition of somatic embryogenesis. Mol Biol Rep 37:2493-2507. doi:10.1007/ s11033-009-9764-3

Kriegs B, Theisen R, Schnabl H (2006) Inositol 1,4,5-trisphosphate and Ran expression during simulated and real microgravity. Protoplasma 229:163-174. doi:10.1007/s00709-006-0214-y

Lai Z, Lin Y (2013) Analysis of the global transcriptome of longan (Dimocarpus longan Lour.) embryogenic callus using illumina paired-end sequencing. BMC Genomics 14:561. doi:10.1186/1471-2164-14-561

Lai ZX, Chen CL, Zeng LH, Chen ZG (2000) Somatic embryogenesis in longan (Dimocarpus longan Lour.). In: Jain SM, Gupta PK, Newton RJ (eds) Somatic embryogenesis in woody plants. Kluwer, Dordrecht, pp 415-431

Lai CC, Lai ZX, Fang ZZ, He Y, Jiang SR (2012) Proteomic analysis of early somatic embryogenesis in Longan (Dimocarpus longan Lour.). Sci Agric Sin 45:1775-1790. doi:10.3864/j.issn.0578-1752.2012.09.013

Lee Y, Kim MH, Kim SK, Kim SH (2008) Phytochrome-mediated differential gene expression of plant Ran/TC4 small G-proteins. Planta 228:215-224. doi:10.1007/s00425-008-0745-X

Lin YL, Lai ZX (2010) Reference gene selection for qPCR analysis during somatic embryogenesis in longan tree. Plant Sci 178:359-365. doi:10.1016/j. plantsci.2010.02.005

Lü S, Fan Y, Jin C (2011) Overexpression of a Ran GTPase homologous gene, FaRan from tall fescue, in transgenic Arabidopsis. Biol Plant 55:331-334. doi:10.1007/s10535-011-0048-0

Ma Q-H (2007) Small GTP-binding proteins and their functions in plants. J Plant Growth Regul 26:369-388. doi:10.1007/s00344-007-9022-7

Mahong B, Roytrakul S, Phaonaklop N, Wongratana J, Yokthongwattana K (2012) Proteomic analysis of a model unicellular green alga, Chlamydomonas reinhardtii, during short-term exposure to irradiance stress reveals significant down regulation of several heat-shock proteins. Planta 235:499-511. doi:10.1007/s00425-011-1521-x 
Matsumoto TK (2006) Genes uniquely expressed in vegetative and potassium chlorate induced floral buds of Dimocarpus longan. Plant Sci 170:500510. doi:10.1016/j.plantsci.2005.09.016

Meier I, Somers DE (2011) Regulation of nucleocytoplasmic trafficking in plants. Curr Opin Plant Biol 14:538-546. doi:10.1016/j.pbi.2011.06.005

Merkle T (2011) Nucleo-cytoplasmic transport of proteins and RNA in plants. Plant Cell Rep 30:153-176. doi:10.1007/s00299-010-0928-3

Michalczuk L, Cooke TJ, Cohen JD (1992a) Auxin levels at different stages of carrot somatic embryogenesis. Phytochemistry 31:1097-1103. doi:10.1016/0031-9422(92)80241-6

Michalczuk L, Ribnicky DM, Cooke TJ, Cohen JD (1992b) Regulation of indole3-acetic acid biosynthetic pathways in carrot cell cultures. Plant Physiol 100:1346-1353

Nackley AG, Shabalina SA, Tchivileva IE, Satterfield K, Korchynskyi O, Makarov SS, Maixner W, Diatchenko L (2006) Human catechol-O-methyltransferase haplotypes modulate protein expression by altering mRNA secondary structure. Science 314:1930-1933. doi:10.1126/science.1131262

Pan Z, Zhu S, Guan R, Deng X (2010) Identification of 2,4-D-responsive proteins in embryogenic callus of Valencia sweet orange (Citrus sinensis Osbeck) following osmotic stress. Plant Cell Tissue Organ Cult 103:145-153. doi:10.1007/s11240-010-9762-0

Plotkin JB, Kudla G (2011) Synonymous but not the same: the causes and consequences of codon bias. Nat Rev Genet 12:32-42. doi:10.1038/nrg2899

Skrzycki M, Majewska M, Czeczot H (2010) Superoxide dismutase mRNA and protein level in human colorectal cancer. Cent Eur J Biol 5:590-599. doi:10.2478/s11535-010-0054-9

Stone SL, Kwong LW, Yee KM, Pelletier J, Lepiniec L, Fischer RL, Goldberg RB, Harada JJ (2001) LEAFY COTYLEDON2 encodes a B3 domain transcription factor that induces embryo development. Proc Natl Acad Sci USA 98:11806-11811. doi:10.1073/pnas.201413498

Stone SL, Braybrook SA, Paula SL, Kwong LW, Meuser J, Pelletier J, Hsieh T-F, Fischer RL, Goldberg RB, Harada JJ (2008) Arabidopsis LEAFY COTYLEDON2 induces maturation traits and auxin activity: implications for somatic embryogenesis. Proc Natl Acad Sci USA 105:3151-3156. doi:10.1073/ pnas.0712364105

Su YH, Zhao XY, Liu YB, Zhang CL, O’Neill SD, Zhang XS (2009) Auxininduced WUS expression is essential for embryonic stem cell renewal during somatic embryogenesis in Arabidopsis. Plant J 59:448-460. doi:10.1111/j.1365-313X.2009.03880.x
Takai Y, Sasaki T, Matozaki T (2001) Small GTP-binding proteins. Physiol Rev 81:153-208

VanBuren R, Li J, Zee F, Zhu J, Liu C, Arumuganathan A, Ming R (2011) Longli is not a hybrid of Longan and Lychee as revealed by genome size analysis and trichome morphology. Trop Plant Biol 4:228-236. doi:10.1007/ s12042-011-9084-3

Vandesompele J, Preter KD, Pattyn F, Poppe B, Roy NV, Paepe AD, Speleman F (2002) Accurate normalization of real-time quantitative RT-PCR data by geometric averaging of multiple internal control genes. Genome Biol 3:research0034.1-research0034.11

Vernoud V, Horton AC, Yang Z, Nielsen E (2003) Analysis of the small GTPase gene superfamily of Arabidopsis. Plant Physiol 131:1191-1208. doi:10.1104/pp.013052

Wang X, Xu Y, Han Y, Bao S, Du J, Yuan M, Xu Z, Chong K (2006) Overexpression of RAN1 in rice and arabidopsis alters primordial meristem, mitotic progress, and sensitivity to auxin. Plant Physiol 140:91-101. doi:10.1104/ pp. 105.071670

Wang H, Wang Q, Pape U, Shen B, Huang J, Wu B, Li X (2010) Systematic investigation of global coordination among mRNA and protein in cellular society. BMC Genomics 11:364

Xu C, Huang B (2008) Root proteomic responses to heat stress in two Agrostis grass species contrasting in heat tolerance. J Exp Bot 59:4183-4194. doi:10.1093/jxb/ern258

Xu C, Huang B (2010) Differential proteomic response to heat stress in thermal Agrostis scabra and heat-sensitive Agrostis stolonifera. Physiol Plant 139:192-204. doi:10.1111/j.1399-3054.2010.01357.x

Yoshimura K, Masuda A, Kuwano M, Yokota A, Akashi K (2008) Programmed proteome response for drought avoidance/tolerance in the root of a C3 Xerophyte (wild watermelon) under water deficits. Plant Cell Physiol 49:226-241. doi:10.1093/pcp/pcm180

Zang A, Xu X, Neill S, Cai W (2010) Overexpression of OsRAN2 in rice and Arabidopsis renders transgenic plants hypersensitive to salinity and osmotic stress. J Exp Bot 61:777-789. doi:10.1093/jxb/erp341

Zheng GM, Xu LX, Wu P, Xie HH, Jiang YM, Chen F, Wei XY (2009) Polyphenols from longan seeds and their radical-scavenging activity. Food Chem 116:433-436. doi:10.1016/j.foodchem.2009.02.059

Zuker M (2003) Mfold web server for nucleic acid folding and hybridization prediction. Nucleic Acids Res 31:3406-3415. doi:10.1093/nar/gkg595

\section{Submit your manuscript to a SpringerOpen ${ }^{\odot}$ journal and benefit from:}

- Convenient online submission

- Rigorous peer review

- Immediate publication on acceptance

- Open access: articles freely available online

- High visibility within the field

- Retaining the copyright to your article

Submit your next manuscript at $>$ springeropen.com 